\title{
Energy-efficient Power Allocation for Point-to-point MIMO Systems over the Rayleigh Fading Channel
}

\author{
Fabien Héliot, Member, IEEE, Muhammad Ali Imran, Member, IEEE, and Rahim Tafazolli, Member, IEEE,
}

\begin{abstract}
It is well-established that transmitting at full power is the most spectral-efficient power allocation strategy for pointto-point (P2P) multi-input multi-output (MIMO) systems, however, can this strategy be energy efficient as well? In this letter, we address the most energy-efficient power allocation policy for symmetric P2P MIMO systems by accurately approximating in closed-form their optimal transmit power when a realistic MIMO power consumption model is considered. In most cases, being energy efficient implies a reduction in transmit and overall consumed powers at the expense of a lower spectral efficiency.
\end{abstract}

Index Terms-MIMO system, Energy efficiency, power allocation, realistic power model.

\section{INTRODUCTION}

In communication, energy efficiency (EE) is a well-studied topic as far as power-limited applications are concerned [1], [2]. In such systems, the energy consumption is clearly a crucial factor. By contrast, EE has received little attention for designing large-scale communication network. However, this is beginning to change under the lead of network operators who have found in EE a way to be more eco-friendly and to reduce their operational costs. As a result, EE is now being revisited for unlimited-power applications such as cellular network [3] and the EE as a criterion is becoming as important as the well-established spectral efficiency (SE) metric for designing the next generation of communication network.

EE being the ratio between transmission rate and consumed power [1], it can be improved by jointly adapting them to the channel conditions. In power-limited systems, where $\mathrm{EE}$ is one of the main design criterion, works in [4], [5] have recently come up with energy-efficient power and rate allocations for saving user equipment (UE) energy. In power-unlimited systems, such as cellular network, where $80 \%$ of the energy is consumed at the base station (BS) site [6], effective resource allocation techniques have been successfully implemented in [7], [8] for improving the EE of BS when all the nodes have single antenna. As far as multi-input multi-output (MIMO) systems are concerned, works in [9], [10] have looked at the EE of closed-loop MIMO systems, i.e. when assuming that channel state information (CSI) is available at the transmitter.

In this letter, we propose an energy-efficient power and rate allocation method for symmetric P2P MIMO systems over the Rayleigh fading channel based solely on statistical CSI knowledge. In this scenario, it is well-known that the SEoptimal power allocation policy is to transmit at maximum

F. Héliot, M.A. Imran, and R. Tafazolli are with the Centre for Communication Systems Research, FEPS, University of Surrey, Guildford GU2 7XH, UK (phone: +44 1483689 492; fax: +44 1483686 011; E-mail F.Heliot@Surrey.ac.uk). The research leading to these results has received funding from the European Commission's Seventh Framework Programme FP7/2007-2013 under grant agreement n²47733-project EARTH. power [11], but can this policy also be EE-optimal? We first discuss the EE framework for MIMO system in Section II, which includes the formulation of the P2P MIMO energy consumption per bit based on a realistic power consumption model (PCM). Using this framework in Section III, we accurately approximate in closed-form the transmit power that minimizes the energy consumption, or equivalently maximizes the EE. Relying on our approximation we provide analytical insights in Section IV, which have been cross-validated by simulation results, on how to energy-efficiently allocate transmit power at the BS as a function of the PCM parameters and channel conditions. Our analysis reveals that transmitting at full power can be both optimal in terms of SE and EE but only in limited scenarios. In most cases, being energy efficient implies a reduction in transmit and overall consumed powers at the expense of a lower SE, which emphasizes the existence of a trade-off between these two metrics. As an application, we compare the EE of a $2 \times 2$ MIMO against a single-input singleoutput (SISO) system; results indicate that EE improvements provided by MIMO over SISO system are mainly the results of SE improvements. Conclusions are drawn in Section V.

\section{MIMO ENERGY EFFICIENCY FRAMEWORK}

The EE of a communication system is closely related to its total power consumption. The total consumed power of a P2P MIMO system, where a BS equipped with $t$ transmit antennas communicates with a UE having $r$ receive antennas, is in effect the sum of the power consumed by these nodes for transmitting and receiving. A comprehensive BS power consumption analysis has recently been undertaken in [3], where realistic PCMs for five types of BS have been defined. This work showed that the relation between the relative radio frequency output power and BS power consumption is nearly linear and, hence, can be linearly approximated. This work also anticipated that DC-DC/AC-DC converter and cooling unit consumed powers do not necessarily grow linearly with the number of antennas. Consequently, refined MIMO BS PCMs have been proposed in [10], [12] by considering that only one part of the overhead power grows linearly with $t$ and one part remains fixed such that the total consumed power is given by

$$
P_{\Sigma}=\Delta_{P} P+t P_{0}+P_{1},
$$

where $P$ is the transmit power, $\Delta_{P}$ is the slope of the PCM, and $P_{0}$ as well as $P_{1}$ are overhead powers. In addition, $P \in\left[0, P_{\max }\right]$ with $P_{\max }$ being the maximum transmit power. For a generic BS, the total overhead power is in the order of hundreds of Watt [3], [12], whereas the UE consumed power is in the order of hundreds of milliwatt [5]. Thus, the latter can be neglected in a P2P setting. 
Relying on the PCM in (1), it has been shown in [12] that the energy consumption per bit, $E_{b}$, over the MIMO Rayleigh flat fading channel can be expressed as

$$
E_{b}=\frac{N}{W C}\left[\Delta_{P} f^{-1}(C)+\frac{t P_{0}+P_{1}}{N}\right],
$$

where $N$ is the noise power, $W(\mathrm{~Hz})$ is the bandwidth and $C(\mathrm{bit} / \mathrm{s} / \mathrm{Hz})$ is the channel capacity per unit bandwidth such that $C=f(P / N)$ with $f(P / N)=\mathrm{E}_{\mathbf{H}}\left\{\log _{2}\left|\mathbf{I}_{r}+\frac{P}{N t} \mathbf{H H}^{\dagger}\right|\right\}$ in the P2P MIMO Rayleigh fading flat channel case [13]. In addition, $f^{-1}(C)$ is the inverse of the MIMO channel capacity per unit bandwidth such that $P=N f^{-1}(C)$, where $f^{-1}(C)$ has been accurately approximated in (15) of [12] for the generic MIMO case. The latter further simplifies as $P / N=$

$$
f^{-1}(C) \approx \frac{1}{4}\left\{-1+\left(1+\left[W_{0}\left(-2^{-\left(\frac{C}{2 t}+1\right)} e^{-\frac{1}{2}}\right)\right]^{-1}\right)^{2}\right\}
$$

in the symmetric MIMO channel [12], i.e. if $t=r$, where $W_{0}$ denotes the real branch of the Lambert function [14]. The Lambert $W$ function satisfies $W(z) e^{W(z)}=z$, with $z \in \mathbb{C}$ [14]. Its real branch, $W_{0}$, is such that $W_{0}:\left[-e^{-1},+\infty\right) \mapsto$ $[-1,+\infty)$ and is monotonically increasing over its domain.

\section{P2P MIMO ENERGY-EFFICIENT Power ALlocation}

Inserting (3) into (2), the energy per bit over the symmetric MIMO channel can be accurately approximated as

$$
E_{b}(X) \approx \alpha \frac{e^{X+1}\left(e^{X+1}-1\right)+\beta}{e^{-(X+1)}+2 X+1},
$$

where $\alpha=\frac{\ln (2) \Delta_{P} N}{W t}, \beta=\frac{t P_{0}+P_{1}}{\Delta_{P} N}$, and $X=$ $\ln \left(-\left[W_{0}\left(-2^{-\left(\frac{C}{2 t}+1\right)} e^{-\frac{1}{2}}\right)\right]^{-1}\right)-\ln (2)-1$. Clearly, $E_{b}(X)$ is differentiable over its domain, i.e. for any $X \in[-1,+\infty]$, such that $\frac{\partial E_{b}(X)}{\partial X}$ can be expressed after simplifications as

$$
\frac{\partial E_{b}(X)}{\partial X} \approx G(X)\left[2 e^{X+1}\left(X e^{X+1}+1\right)-\beta\right],
$$

where $G(X)=\frac{\alpha e^{(X+1)}\left(2 e^{X+1}-1\right)}{\left(e^{X+1}(2 X+1)+1\right)^{2}}>0$. Let $X^{\star}$ be a solution of the equation $\frac{\partial E_{b}(X)}{\partial X}=0$. Then, $\frac{\partial E_{b}(X)}{\partial X} \leq 0$ and $\frac{\partial E_{b}(X)}{\partial X} \geq 0$ for any $X \in\left[-1, X^{\star}\right]$ and $X \in\left[X^{\star},+\infty\right]$, respectively, which in turn implies that $E_{b}(X)$ decreases over $X \in\left[-1, X^{\star}\right]$ and then increases over $X \in\left[X^{\star},+\infty\right]$. Consequently, $E_{b}(X)$ has a unique minimum, which occurs at $X=X^{\star}$. Setting $\frac{\partial E_{b}\left(X=X^{\star}\right)}{\partial X}=0$ in (5), we obtain that

$$
g\left(X^{\star}\right)=2 e^{X^{\star}+2}\left(X^{\star} e^{X^{\star}}+e^{-1}\right) \approx \beta,
$$

which unfortunately cannot be solved in closed-form, but through a line-search algorithm such as the Newton-Raphson method. However, $g\left(X^{\star}\right)$ in (6) can be simplified for certain ranges of $X^{\star}$ values such that $X^{\star}$ can be accurately approximated in closed-form. For instance, if $X^{\star} e^{X^{\star}} \gg e^{-1}$, then $X^{\star} e^{X^{\star}}+e^{-1} \approx X^{\star} e^{X^{\star}}$ such that $g\left(X^{\star}\right) \approx \widetilde{g}_{0}\left(X^{\star}\right)=$ $2 X^{\star} e^{2 X^{\star}+2}$. It can be noticed that the relative approximation error between $g$ and $\widetilde{g}_{0}$ that is defined as

$$
\eta\left(\widetilde{g}_{0}\right)=100 \%\left(\left|g(X)-\widetilde{g}_{0}(X)\right| /|g(X)|\right)
$$

TABLE I

ACCURATE APPROXIMATIONS OF $\beta$ AND $X^{\star}$ FOR VARIOUS RANGES OF $X^{\star}$ VALUES

\begin{tabular}{|c|c|c|c|c|}
\hline$X^{\star}$ & $n$ & $\beta \approx \widetilde{g}_{n}\left(X^{\star}\right)=$ & $X^{\star} \approx \widetilde{g}_{n}^{-1}(\beta)=$ & $\eta_{\max }\left(\widetilde{g}_{n}\right)$ \\
\hline$\geq 2.44$ & 0 & $2 X^{\star} e^{2 X^{\star}+2}$ & $\frac{1}{2} W_{0}\left(\beta e^{-2}\right)$ & $<1.3 \%$ \\
\hline $\begin{array}{c}<.44 \\
\& \geq 0\end{array}$ & 1 & $2\left(a X^{\star}+1\right) e^{b X^{\star}+1}$ & $\frac{1}{b} W_{0}\left(\frac{\beta b}{2 a} e^{\frac{b-a}{a}}\right)-\frac{1}{a}$ & $<1.3 \%$ \\
\hline $\begin{array}{l}0<\& \\
\geq-1\end{array}$ & 2 & $2 \frac{\left(X^{\star}+1\right)^{2}}{1-\left(X^{\star}\right)^{3}} e^{\frac{\left(X^{\star}+1\right)^{2}}{1-\left(X^{\star}\right)^{3}}}$ & $-\frac{1}{3}\left(3+\sqrt[3]{\frac{1}{2}[A(\zeta)+9 B(\zeta)]}\right.$ & $<4.2 \%$ \\
\hline$\sim-1$ & 3 & $\left(X^{\star}+1\right)^{2}$ & $\left.+\zeta+\sqrt[3]{\frac{1}{2}[A(\zeta)-9 B(\zeta)]}\right)$ & \\
\hline
\end{tabular}

is always below $1 \%$ as long as $X^{\star} \geq 2.636$, i.e.

$$
\eta_{\max }\left(\widetilde{g}_{0}\right)=\max _{X^{\star} \in[2.636,+\infty]} \eta\left(\widetilde{g}_{0}\right)<1 \% .
$$

Moreover, $g$ can be well approximated by functions of the form $\widetilde{g}_{1}(X)=2(a X+1) e^{b X+1}$ when $X^{\star} \in[0,3]$. By carefully selecting the parameters $a$ and $b$ such that

$$
\begin{aligned}
& \min _{a, b} \max _{X^{\star}}\left\{\eta\left(\widetilde{g}_{1}\right)\right\} \\
& \text { s.t. } \quad X^{\star} \in[0,3],
\end{aligned}
$$

the approximation error between $g$ and $\widetilde{g}_{1}$ can be kept below $2 \%$ for any $X^{\star} \in[0,3]$. Note that $\eta_{\max }\left(\widetilde{g}_{0}\right)$ decreases as $X^{\star}$ increases, whereas, $\eta_{\max }\left(\widetilde{g}_{1}\right)$ decreases as $X^{\star}$ decreases. Thus, in an effort to harmonize the maximum of the approximation error over the range $X^{\star} \in[0,+\infty]$, we obtain the $X^{\star}$ value for which $\eta_{\max }\left(\widetilde{g}_{0}\right) \simeq \eta_{\max }\left(\widetilde{g}_{1}\right)$, which corresponds to $X^{\star}=2.44, a=1.7577$ and $b=2.1034$ such that $\eta_{\max }\left(\widetilde{g}_{0}\right) \simeq \eta_{\max }\left(\widetilde{g}_{1}\right)<1.3 \%$ for $X^{\star} \in[0,+\infty]$. At the other end of the $X^{\star}$ range of values, $g\left(X^{\star}\right) \approx \widetilde{g}_{3}\left(X^{\star}\right)$ (see Table I) when $X^{\star}$ goes towards -1 , such that $g$ and $\widetilde{g}_{3}$ differs by less than $1 \%$, as long as $X^{\star} \leq-0.985$. Knowing that $g\left(X^{\star}\right) \approx g_{3}\left(X^{\star}\right)$ for $X \sim-1$ and $g\left(X^{\star}\right) \simeq e^{-1}$ for $X^{\star} \rightarrow 0$, we come up with $\widetilde{g}_{2}\left(X^{\star}\right)$ in Table I for accurately approximating $g$ over $X^{\star} \in[-1,0]$ such that $\eta_{\max }\left(\widetilde{g}_{2}\right)<4.2 \%$ for $X^{\star} \in[-1,0]$. Note that $\widetilde{g}_{2}$ reverts to $\widetilde{g}_{3}$ for $X^{\star} \sim-1$.

Replacing $g$ by $\widetilde{g}_{0}$ in (6), the latter can be reformulated as

$$
2 X^{\star} e^{2 X^{\star}} \approx \beta e^{-2},
$$

which can be solved in a straightforward manner by means of the Lambert $\mathrm{W}$ function, such that

$$
X^{\star} \approx \widetilde{g}_{0}^{-1}(\beta)=\frac{1}{2} W_{0}\left(\beta e^{-2}\right)
$$

for $X^{\star} \in[2.44,+\infty]$. Similarly, substituting $g$ with $\widetilde{g}_{1}$ or $\widetilde{g}_{2}$ in (6), we can accurately approximate $X^{\star}$ in closed-form, as it is summarized in Table I. Note that $\zeta=-3+W_{0}(\beta / 2)^{-1}$, $A(\zeta)=2 \zeta^{3}-27(2+\zeta)$ and $B(\zeta)=\left(-\frac{8}{3} \zeta^{3}-3 \zeta^{2}+36 \zeta+\right.$ $72)^{\frac{1}{2}}$ in Table I. Finally, the optimal transmit power $P^{\star}$ can be approximated as

$$
P^{\star} \approx \min \left\{N\left[e^{X^{\star}+1}\left(e^{X^{\star}+1}-1\right)\right], P_{\max }\right\},
$$

since $P \in\left[0, P_{\max }\right]$ and $P \approx N\left[e^{X+1}\left(e^{X+1}-1\right)\right]$. 


\section{Analytical Insights \& Numerical Results}

\section{A. Analytical insights}

Accurately approximating $P^{\star}$ is not only useful for evaluating $P^{\star}$ in a faster way than with a line search algorithm, but when used in conjunction with (6), it is also useful for getting valuable analytical insights on the behavior of $P^{\star}$ as a function of the parameter $\beta$, i.e. $N, t, \Delta_{P}, P_{0}$ and $P_{1}$.

Equation (6) clearly indicates that $X^{\star}$ decreases or increases as $\beta$ decreases or increases, which in turn have some implications on the behavior of $P^{\star}$. For instance if $\beta$ goes to zero then $X^{\star}$ would decrease towards -1 such that $X^{\star} \stackrel{\beta \rightarrow 0}{\sim} \widetilde{g}_{3}^{-1}(\beta)$ (See Table I). Moreover, $N\left[e^{X^{\star}+1}\left(e^{X^{\star}+1}-1\right)\right] \stackrel{X^{\star}}{\sim}{ }^{-1}$ $N\left(X^{\star}+1\right)$ in (12). Consequently,

$$
P^{\star} \stackrel{\beta \rightarrow 0}{\sim} \min \left\{\sqrt{\frac{N\left(t P_{0}+P_{1}\right)}{\Delta_{P}}}, P_{\max }\right\}
$$

for small values of $\beta$. Considering either $N,\left(t P_{0}+P_{1}\right)$ or $\Delta_{P}$ as a variable and keeping the two other parameters fixed, $\beta \rightarrow 0$ implies that either $N$ or $\Delta_{P}$ increases towards infinity, or $\left(t P_{0}+P_{1}\right)$ decreases towards zero, which leads us to the following conclusions:

- As the average noise power $N$ increases and the channel condition worsens, more transmit power is needed to be energy efficient, and $\lim _{N \rightarrow+\infty} P^{\star}=P_{\text {max }}$.

- If the fixed transmit power $t P_{0}+P_{1} \rightarrow 0$, then $P^{\star} \rightarrow 0$ according to (13), which is consistent with the fact that $E_{b}$ is minimized for $P=C=0$ in the idealistic PCM [1], i.e. when $P_{0}=P_{1}=0$ and $\Delta_{P}=1$.

- If the amplifier inefficiency, $\Delta_{P}$, increases towards infinity, then $P^{\star}$ goes to zero because the inefficiency is so severe that it is preferable to avoid transmission.

Conversely, in the case that $\beta$ increases towards infinity, then $X^{\star}$ would also increase towards infinity such that $X^{\star} \stackrel{\beta \rightarrow+\infty}{\sim}$ $\widetilde{g}_{0}^{-1}(\beta)$ (See Table 1$)$ and $N\left[e^{X^{\star}+1}\left(e^{X^{\star}+1}-1\right)\right]^{X^{\star}} \vec{\sim}^{+\infty}$ $N e^{2 X^{\star}+2}$ in (12). Furthermore, we know from [15] that $W_{0}(x)$ can be lower and upper bounded as

$\ln (x)-\ln (\ln (x)) \leq W_{0}(x) \leq \ln (x)-\ln (\ln (x))+\ln \left(1+e^{-1}\right)$

for $x>e^{1}$, which in turn implies that $N e^{2 X^{\star}+2}$ can be lower and upper bounded as

$$
\frac{t P_{0}+P_{1}}{\Delta_{P} \ln \left(\beta e^{-2}\right)} \lesssim N e^{2 X^{\star}+2} \lesssim \frac{\left(1+e^{-1}\right)\left(t P_{0}+P_{1}\right)}{\Delta_{P} \ln \left(\beta e^{-2}\right)}
$$

by inserting $\widetilde{g}_{0}^{-1}(\beta)$ in $N e^{2 X^{\star}+2}$ and then using (14) to bound the latter. Knowing that $\beta \rightarrow+\infty$, it implies that either $N$ decreases towards zero or $\left(t P_{0}+P_{1}\right)$ increases towards infinity since $\Delta_{P} \in[1,+\infty]$, which leads us to the following conclusions:

- As $N$ decreases, less transmit power is needed to transmit data in an energy-efficient manner such that $\lim _{N \rightarrow 0} P^{\star}=0$, since $\lim _{N \rightarrow 0} 1 / \ln (\beta)=0$ in (15).

- In the case that $t P_{0}+P_{1} \gg \Delta_{P} P_{\max }, P_{\Sigma}$ in (1) becomes in effect independent of $P$ and minimizing the energy consumption per bit is then equivalent to maximizing the channel capacity, which is obviously maximized for $P^{\star}=$ $P_{\max }$. Thus, whenever $\Delta_{P}$ is fixed and $t P_{0}+P_{1}$ increases towards infinity, then $P^{\star}$ increases towards $P_{\max }$.

\section{B. Numerical Results}

In Table I, we have obtained accurate approximations of $X^{\star}$ for three range of $X^{\star}$ values. Thus, we need the following procedure for obtaining $P^{\star}$ via our expressions in Table I:

1) Compute $\widetilde{X}^{\star}=\widetilde{g}_{0}^{-1}(\beta)$;

2) if $\widetilde{X}^{\star}<2.44$, then compute $\widetilde{X}^{\star}=\widetilde{g}_{1}^{-1}(\beta)$;

3) if $\widetilde{X}^{\star}<0$, then compute $\widetilde{X}^{\star}=\widetilde{g}_{2}^{-1}(\beta)$;

4) $P^{\star} \approx \widetilde{P}^{\star}=\min \left\{N\left[e^{\widetilde{X}^{\star}+1}\left(e^{\widetilde{X}^{\star}+1}-1\right)\right], P_{\max }\right\}$.

Note also that (6) is based on an approximation, i.e. equation (3), whereas, $f^{-1}(C)$ can only be obtained numerically. Having obtained $f^{-1}(C)$ numerically via Telatar formulation of $f(P / N)$ in [13], we have plotted in Fig. 1 the exact $P^{\star}$ by using a time-consuming exhaustive search for finding the minimum of (2). This approach acts here as a benchmark.

In order to show the accuracy of $\widetilde{P}^{\star}$, we compare in Fig. 1 the exact $P^{\star}$ with $P^{\star}$ obtained via a line-search on equation (6) and $\widetilde{P}^{\star}$ as a function of $N$ and $\frac{t P_{0}+P_{1}}{\Delta_{P}}$. We consider that $P_{\max }=40 \mathrm{~W}$ and use the PCM values of [12], i.e. $\Delta_{P}=$ $7.25, t=2, P_{0}=244 \mathrm{~W}$ and $P_{1}=225 \mathrm{~W}$ in the upper part of the graph and $N=1$ in lower part of the graph. Results clearly indicate the great accuracy of our procedure based on closed-form approximations of $X^{\star}$. Indeed, $\widetilde{P}^{\star}$ differs from the exact $P^{\star}$ by at most $1.1 \%$ and $2.1 \%$ in the upper and lower parts of the graph, respectively. These results also confirm our analytical insights of Section IV-A, i.e. $P^{\star}$ increases as the noise power increases and as $t P_{0}+P_{1}$ increases or $\Delta_{P}$ decreases.

As an application for our energy-efficient power allocation method, we compare in Fig. 2 the EE of a 2x2 MIMO system against the EE of a SISO system for different scenarios. Let us define the EE gain between MIMO and SISO systems as

$$
G_{\mathrm{EE}}=\frac{E_{b, \mathrm{SISO}}}{E_{b, \mathrm{MIMO}}}=\frac{C_{\mathrm{MIMO}}\left(\Delta_{P} P_{\mathrm{SISO}}+P_{0}+P_{1}\right)}{C_{\mathrm{SISO}}\left(\Delta_{P} P_{\mathrm{MIMO}}+t P_{0}+P_{1}\right)}
$$

when assuming that both systems have the same power model and bandwidth. An increase in $G_{\mathrm{EE}}$ can result solely from an increase of SE (MIMO providing a better SE than SISO for $P_{\text {SISO }}=P_{\text {MIMO }}$ ), solely from a decrease in consumed power (MIMO consuming less power than SISO for $C_{\text {SISO }}=C_{\text {MIMO }}$ ) or a combination of both. The former type of $\mathrm{EE}$ gain is actually equivalent to a scaled SE gain, $G_{\mathrm{EE}, \mathrm{SE}}$, and MIMO is already well-known to be very effective for improving the SE, whereas we have investigated the second type of $\mathrm{EE}$ gain, i.e. the MIMO vs. SISO EE gain due solely to power reduction, $G_{\mathrm{EE}, \mathrm{PR}}$, in [12]. Here, we use our approximation of $P^{\star}$ for evaluating the maximum achievable MIMO vs. SISO EE gain, $G_{\mathrm{EE}, P^{\star}}$, and compare it against $G_{\mathrm{EE}, \mathrm{SE}}$ and $G_{\mathrm{EE}, \mathrm{PR}}$. Figure 2 depicts each EE gain and their respective total consumed power and $\mathrm{SE}$ as a function of the noise power $N$ for $P_{\text {max }}=40 \mathrm{~W}, \Delta_{P}=7.25, t=2, P_{0}=244 \mathrm{~W}$ and $P_{1}=225$ $\mathrm{W}$. In order to compute the EE gains, we assume that the SISO system transmit with either a SE-optimal or EE-optimal power, i.e. $P_{\text {SISO }}=P_{\max }$ or $P_{\text {SISO }}=P_{\text {SISO }}^{\star}$. Focusing on the 


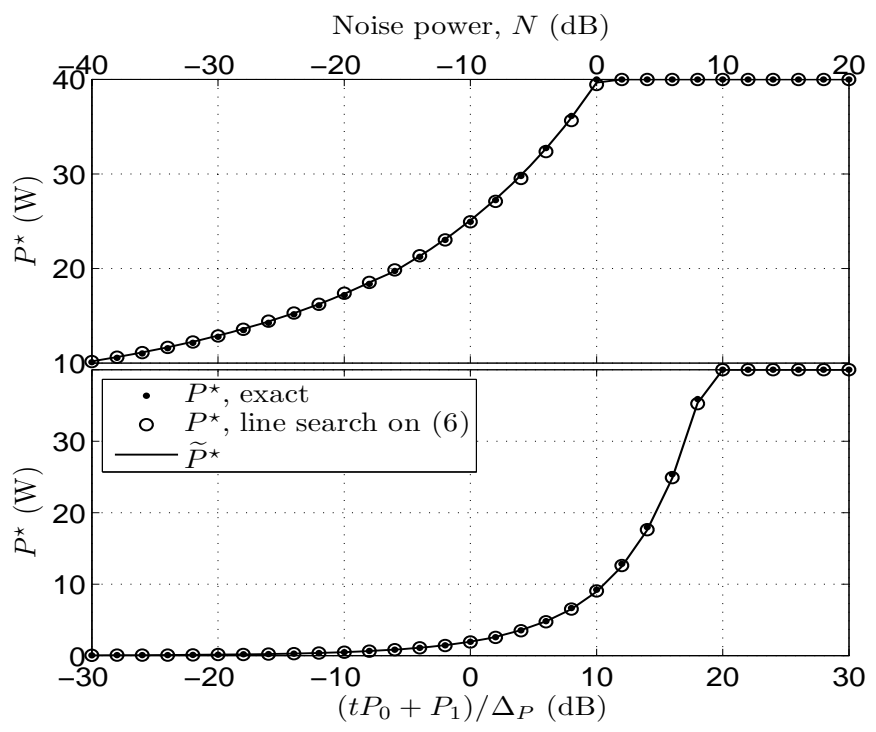

Fig. 1. Comparison of different approaches for obtaining $P^{\star}$.

$P_{\text {SISO }}=P_{\max }$ case, the results first indicate that for $N>-2$ $\mathrm{dB}$, a $2 \times 2$ MIMO system cannot be more energy efficient than SISO solely via power reduction and $G_{\mathrm{EE}, P^{\star}}=G_{\mathrm{EE}, \mathrm{SE}}$. Thus, a $2 \times 2$ MIMO system can paradoxically be more energy efficient than SISO even though it consumes more power. For low $N$ values, $\mathrm{EE}$ can be achieved solely via power saving such that $2 \times 2$ MIMO can be up to $6 \%$ more energy efficient than SISO but a the expense of a more than halved SE. Whereas, the maximum EE gain is also achieved by tradingoff SE for power but in a more balanced way. For instance at $N=-30 \mathrm{~dB}, G_{\mathrm{EE}, P \star}$ is obtained when increasing the total consumed power by $90 \mathrm{~W}$ in comparison with $G_{\mathrm{EE}, \mathrm{PR}}$ and incurs a $3 \mathrm{bit} / \mathrm{s} / \mathrm{Hz}$ loss in SE in comparison with $G_{\mathrm{EE}, \mathrm{SE}}$, such that $G_{\mathrm{EE}, P \star}$ is $10 \%$ and $53 \%$ higher than $G_{\mathrm{EE}, \mathrm{SE}}$ and $G_{\mathrm{EE}, \mathrm{PR}}$, respectively.

In the case that both the $2 \times 2$ MIMO and SISO systems use an EE-optimal power allocation, SISO system always consume less power than MIMO and the EE gains decrease with $N$ since the SISO total consumed power starts to decrease for higher values of $N$ than MIMO. These results confirm that for this particular set of PCM parameters the EE improvement provided by MIMO is mainly the results of SE improvement.

\section{CONCLUSION}

In this letter, we have addressed the most energy-efficient power allocation policy for $\mathrm{P} 2 \mathrm{P}$ MIMO when considering a realistic MIMO power model consumption (PCM) by accurately approximating in closed-form the transmit power that minimizes the energy consumption per bit of P2P MIMO. Based on the analytical insights gained from our approximation, we have revealed that transmitting at full power, which is optimal in terms of SE, is only optimal in terms of EE for limited scenarios. In most cases, being energy efficient implies a reduction in transmit and overall consumed powers at the expense of a lower SE, which emphasizes the existence of a trade-off between these two metrics. Balancing this trade-off

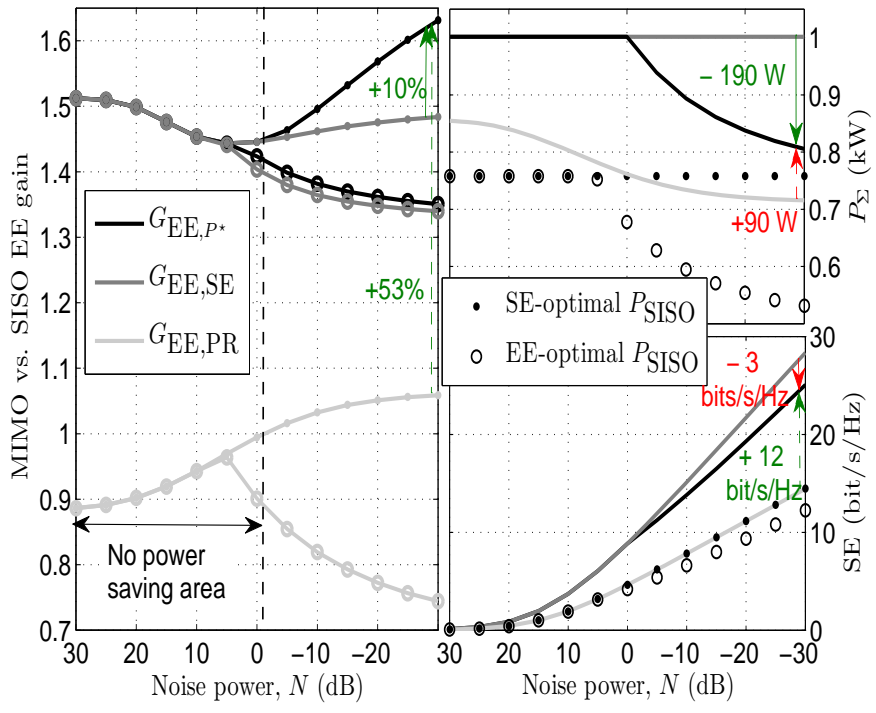

Fig. 2. $2 \times 2$ MIMO vs. SISO EE gains and their respective SEs and total consumed powers.

is the key to maximize the potential benefit in terms of $\mathrm{EE}$ of using a $2 \times 2$ MIMO instead of a SISO system.

\section{REFERENCES}

[1] H. M. Kwon and T. G. Birdsall, "Channel Capacity in Bits per Joule," IEEE J. Ocean. Eng., vol. OE-11, no. 1, pp. 97-99, Jan. 1986.

[2] S. Cui, A. J. Goldsmith, and A. Bahai, "Energy-Efficiency of MIMO and Cooperative MIMO Techniques in Sensor Networks," IEEE J. Sel. Areas Commun., vol. 22, no. 6, pp. 1089-1098, Aug. 2004.

[3] G. Auer et al., "How Much Energy is Needed to Run a Wireless Network ?" IEEE Wireless Commun. Mag., vol. 18, no. 5, pp. 40-49, Oct. 2011.

[4] F. Meshkati et al., "An Energy-Efficient Approach to Power Control and Receiver Design in Wireless Networks," IEEE Trans. Commun., vol. 5, no. 1, pp. 3306-3315, Nov. 2006.

[5] G. Miao, N. Himayat, and G. Y. Li, "Energy-Efficient Link Adaptation in Frequency-Selective Channels," IEEE Trans. Commun., vol. 58, no. 2, pp. 545-554, Feb. 2010.

[6] A. Fehske, G. P. Fettweis, J. Malmodin, and G. Biczok, "The Global Carbon Footprint of Mobile Communiations: The Ecological And Economic Perspective," vol. 49, no. 8, pp. 55-62, Aug. 2011.

[7] Z. Chong and E. Jorswieck, "Analytical Foundation for Energy Efficiency Optimisation in Cellular Networks with Elastic Traffic," in Proc. MOBILIGHT 2011, Bilao, Spain, May 2011.

[8] F. Héliot, M. A. Imran, and R. Tafazolli, "Energy-Efficiency based Resource Allocation for the Scalar Broadcast Channel," in Proc. IEEE WCNC, Paris, France, Apr. 2012.

[9] R. S. Prabhu and B. Daneshrad, "Energy-Efficient Power Loading for a MIMO-SVD System and Its Performance in Flat Fading," in Proc. IEEE Globecom, Miami, USA, Dec. 2010.

[10] J. Xu, L. Qiu, and C. Yu, "Improving energy efficiency through multimode transmission in the downlink MIMO systems," EURASIP Journal on Wireless Communications and Networking, 2011, 2011:200.

[11] G. J. Foschini and M. J. Gans, "On Limits of Wireless Communications in a Fading Environment when using Multiple Antennas," Wireless Personal Commun., vol. 6, pp. 311-335, 1998.

[12] F. Héliot, M. A. Imran, and R. Tafazolli, "On the Energy EfficiencySpectral Efficiency Trade-off over the MIMO Rayleigh Fading Channel," IEEE Trans. Commun., to appear in 2012, (early access: http://ieeexplore.iee.org)

[13] I. E. Telatar, "Capacity of Multi-antenna Gaussian Channels," Europ. Trans. Telecommun. and Related Technol., vol. 10, no. 6, pp. 585-596, Nov. 1999.

[14] R. M. Corless, G. H. Gonnet, D. E. G. Hare, D. J. Jeffrey, and D. E. Knuth, "On the LambertW Function," Adv. Comput. Math., vol. 5, pp. 329-359, 1996.

[15] A. Hoorfar and M. Hassani, "Inequalities on the Lambert W Function and Hyperpower Function," J. Inequal. Pure and Appl. Math., vol. 9, no. 2, Mar. 2008. 